\title{
NORM INEQUALITIES FOR OFF-CENTERED MAXIMAL OPERATORS
}

\author{
RICHARO L. WHEEDEN ${ }^{1}$
}

\begin{abstract}
Sufficient conditions are derived in order that there exist strongtype weighted norm incqualities for some off-centered maximal functions. The maximal functions are of Hardy-Littlewood and fractional types taken over starlike sets in $\mathbb{R}^{n}$. The sufficient conditions are close to necessary and extend some previously known weak-type results.
\end{abstract}

\section{Introduction}

In [CWW], weighted norm inequalities are derived for some integral and maximal operators associated with starlike sets in Euclidean space $\mathbb{R}^{n}$. Our aim now is to extend the results which deal with analogues of the Hardy-Littlewood and fractional maximal functions. The situation we will consider is closely adapted to both the geometry of the sets used in the definitions of these functions and to the relationship between these sets and the point at which the maximal functions are formed. The study of averages of functions over sets other than balls or cubes has a long history, and for some other weighted results we refer to $[\mathrm{J}]$ and [P3], and the references cited there.

To be more precise, given $0 \leq \mu<n$ and two (possibly unrelated) sets $S$ and $E$ in $\mathbb{R}^{n}$, we consider the maximal function

$$
\mathcal{M}_{S, E, \mu} f(x)=\sup _{\substack{t>0, z \in \mathbb{R}^{n} \\ x \in z+t E}} t^{\mu-n} \int_{z+t S}|f(y)| d y,
$$

where $z+t E$ denotes the set $\{z+t \xi: \xi \in E\}$, and similarly for $z+t S$. We are most interested in the case when $S$ is starlike about the origin and $E$ is bounded. If $\mu=0,(1.1)$ is a special case of an operator considered

${ }^{1}$ Supported in part by NSF grant DMS91-04195. 
in [Cor], although the normalizations are different, and for $0 \leq \mu<n$ it was studied in [CWW]. A simple example occurs by writing $x=\left(x^{\prime}, x_{n}\right)$ with $x^{\prime}=\left(x_{1}, \ldots, x_{n-1}\right)$ and choosing $E$ to be the unit cube $Q_{1}$ centered at 0 and $S$ to be the unbounded set

$$
S_{\gamma}=\left\{x:\left|x_{n}\right| \leq \min \left\{1, \frac{c_{0}}{\left|x^{\prime}\right| \gamma}\right\}\right\}, \quad \gamma>0 .
$$

If we pick $c_{0}$ so that $Q_{1} \subset S_{\gamma}$, then the requirement in (1.1) that $x \in$ $z+t E$ simply amounts to requiring that $x$ belong to the cube-like central portion of $z+t S_{\gamma}$ as opposed to lying farther out in the "skirt" of $z+t S_{\gamma}$.

The weak-type behavior of (1.1) was described in [CWW], and we now want to study its strong-type behavior. For purposes of comparison, we define the centered maximal function

$$
\mathcal{M}_{S, \mu} f(x)=\sup _{t>0} t^{\mu-n} \int_{x+t S}|f(y)| d y, \quad 0 \leq \mu<n,
$$

which corresponds to choosing $E$ to be the empty set in (1.1). Both weak and strong-type results for (1.3) are derived in [CWW], and to put our results in perspective, we recall these for the model case $S=S_{\gamma}$ given in (1.2). For $a \geq 1$ and fixed $\gamma>0$, we associate the linear operators $\delta_{a} x=\left(a x_{1}, \ldots, a x_{n-1}, a^{-\gamma} x_{n}\right)$ and the rectangles $R_{a}=\delta_{a} Q_{1}$. These rectangles are naturally adapted to $S_{\gamma}$ since

$$
\bigcup_{a \geq 1} R_{a} \subset S_{\gamma} \subset \bigcup_{j=0}^{\infty} \kappa R_{2^{j}}
$$

for some geometric constant $\kappa$. Given a rectangle $R$, we denote by $\mathcal{B}(R)$ the collection of all translates and dilates of $R$, i.e.,

$$
\mathcal{B}(R)=\left\{z+t R: z \in \mathbb{R}^{n}, t>0\right\} .
$$

Let $1<p \leq q<\infty, p^{\prime}=p /(p-1)$, and $w(x), v(x)$ be nonnegative locally intcgrable weight functions. Denote $\sigma=v^{-1 /(p-1)}$. It is proved in [CWW] that if the centered maximal operator $\mathcal{M}_{S_{\gamma, \mu}}$ satisfies the weak-type estimate

$$
w\left\{x: \mathcal{M}_{S_{\gamma}, \mu} f(x)>\lambda\right\} \leq\left(\frac{c\|f\|_{p, v}}{\lambda}\right)^{q},
$$

with $c$ independent of $f$ and $\lambda, \lambda>0$, then

$$
|R|^{\frac{\mu}{n}-1} w(R)^{\frac{1}{q}} \sigma(R)^{\frac{1}{p^{\prime}}} \leq C\left|R_{a}\right|^{\frac{\mu}{n}-1}
$$


for all $R \in \mathcal{B}\left(R_{a}\right)$ and all $a \geq 1$. Here we have used the standard notations $w(A)=\int_{A} w d x$,

$$
\|f\|_{p, v}=\left(\int_{\mathbb{R}^{n}}|f(x)|^{p} v(x) d x\right)^{\frac{1}{p}},
$$

and $c$ for a constant which may be different at different occurrences. Conversely, the weak-type estimate holds if there exists a monotone function $C(a), a \geq 1$, such that

$$
|R|^{\frac{\mu}{n}-1} w(R)^{\frac{1}{a}} \sigma(R)^{\frac{1}{p^{\prime}}} \leq C(a)\left|R_{a}\right|^{\frac{\mu}{n}-1}, \quad R \in \mathcal{B}\left(R_{a}\right)
$$

and

$$
\int_{1}^{\infty} C(a) \frac{d a}{a}<\infty
$$

Moreover, we have the strong-type estimate

$$
\left\|\mathcal{M}_{S_{\gamma, \mu}} f\right\|_{q, w} \leq c\|f\|_{p, v},
$$

$1<p \leq q<\infty$, if there exists $r>1$ so that

$$
|R|^{\frac{\mu}{n}-\frac{1}{p}} w(R)^{\frac{1}{q}}\left(\frac{1}{|R|} \int_{R} \sigma^{r} d x\right)^{\frac{1}{r}} \leq C(a)\left|R_{a}\right|^{\frac{\mu}{n}-1}
$$

for all $R \in \mathcal{B}\left(R_{a}\right), a \geq 1$, and $C(a)$ is a monotone function which satisfies (1.5). Of course, (1.6) is stronger than (1.4) due to Hölder's inequality.

For the uncentered operator (1.1), only a weak-type estimate is proved in $[\mathrm{CWW}]$. To describe it, let $\delta_{a}^{*}$ be defined by $\delta_{a}^{*}(x)=$ $\left(a x_{1}, \ldots, a x_{n-1}, x_{n}\right)$ for $a \geq 1$, and let $R_{a}^{*}=\delta_{a}^{*} Q_{1}$. Note for future reference that $R_{a}^{*}$ is the smallest rectangle which contains both $R_{a}$ and $Q_{1}$. To each $R \in \mathcal{B}\left(R_{a}\right)$, associate a rectangle $R^{*}$ as follows: if $R=z+t R_{a}$, then $R^{*}=z+t R_{a}^{*}$. Thus the pair $\left(R, R^{*}\right)$ is a joint translation and dilation of $\left(R_{a}, R_{a}^{*}\right)$ by the same $z, t$. It is proved in [CWW] that if $1<p \leq q<\infty$ and

$$
w\left\{x: \mathcal{M}_{S_{\gamma}, Q_{1}, \mu} f(x)>\lambda\right\} \leq\left(\frac{C\|f\|_{p, v}}{\lambda}\right)^{q},
$$

then

$$
|R|^{\frac{\mu}{2}-1} w\left(R^{*}\right)^{\frac{1}{q}} \sigma(R)^{\frac{1}{\nu^{\prime}}} \leq C\left|R_{a}\right|^{\frac{\mu}{n}-1}
$$


for all pairs $\left(R, R^{*}\right), R \in \mathcal{B}\left(R_{a}\right)$, and all $a \geq 1$. Conversely, suppose $1<p \leq q<\infty$ and there is a monotone function $C(a)$ such that

$$
|R|^{\frac{\mu}{n}-1} w\left(R^{*}\right)^{\frac{1}{a}} \sigma(R)^{\frac{1}{p^{\prime}}} \leq C(a)\left|R_{a}\right|^{\frac{2}{n}-1}
$$

for all $R \in \mathcal{B}\left(R_{a}\right)$ and all $a \geq 1$. If $C(a)$ also satisfies (1.5) then the weak-type estimate (1.7) holds.

Even in the unweighted case $w=v=1$, it follows that the results for the centered and uncentered maximal operators associated with $S_{\gamma}$ are different. In fact, it is easy to check that the conditions then require $1 / q=1 / p-\mu / n$, that the centered maximal function is strong-type for $\gamma>n-1$ if $I<p<n / \mu$, but that even weak-type for the uncentered maximal function requires

$$
p \geq \frac{\gamma}{\gamma-(n-1)\left(1-\frac{\mu}{n}\right)}(>1)
$$

a positive result being guaranteed when strict inequality holds.

For the model case $S=S_{\gamma}$, we will prove the following strong-type result for (1.1).

Theorem 1. Let $\gamma>0$ and $S_{\gamma}$ be defined by (1.2). Let $0 \leq \mu<n$, $1<p \leq q<\infty$ and assume there exists $r>1$ so that

$$
|R|^{\frac{\mu}{n}-\frac{1}{p}} w\left(R^{*}\right)^{\frac{1}{q}}\left(\frac{1}{|R|} \int_{R} \sigma^{r} d x\right)^{\frac{1}{\gamma p^{\prime}}} \leq C(a)\left|R_{a}\right|^{\frac{\mu}{n}-1}
$$

for all $R \in \mathcal{B}\left(R_{0}\right)$ and all $a \geq 1$, where $C(a)$ is a monotone function which satisfies (1.5). Then

$$
\left\|\mathcal{M}_{S_{\gamma}, Q_{1, \mu}} f\right\|_{q, w} \leq c\|f\|_{p, v} .
$$

A result for gencral starlike $S$ is given in Section 3 . Condition (1.8) is analogous to (1.6) for the centered maximal function.

To prove Theorem 1 , we use a covering technique given by C. P. Calderón in $[\mathrm{Ca}]$ together with a result we now describe. Let $\mathcal{B}$ be the family of all translates and dilates of a fixed rectangle $R_{B}$ (i.e., $\mathcal{B}=\mathcal{B}\left(R_{B}\right)$ in our previous notation). Of course $R_{B}$ is not uniquely determined by $B$ but its eccentricities (ratios of edgelengths) are, and we may assume without loss of generality that its first edgelength is 1 . Thus, for example, we may view the basic rectangle in $B\left(R_{b}\right)$ as having edgelengths $1, \ldots, 1, a^{-\gamma-1}$ rather than $a, \ldots, a, a^{-\gamma}$. To each $R \in \mathcal{B}$, 
associate a set (not necessarily a rectangle) $R^{*}$ so that the following holds:

$$
\text { If } R_{1}, R_{2} \in \mathcal{B} \text { and } R_{1} \subset R_{2} \text { then } R_{1}^{*} \subset R_{2}^{*} \text {. }
$$

For example, the pairs $\left(R, R^{*}\right)$ of joint translates and dilates of $\left(R_{a}, R_{a}^{*}\right)$ defined earlier have this property. More generally, if $R_{\mathcal{B}}^{*}$ is defined to be any rectangle containing $R_{\mathcal{B}}$, and given $R \in \mathcal{B}, R=z+t R_{\mathcal{B}}$, we define $R^{*}=z+t R_{B}^{*}$ then the pairs $\left(R, R^{*}\right)$ satisfy (1.9).

For such a collection of pairs and $0 \leq \alpha<1$, define

$$
M_{\alpha} f(x)=\sup _{\substack{R \in \mathcal{B} \\ R^{*} \ni x}}|R|^{\alpha-1} \int_{R}|f(y)| d y .
$$

Of course this depends on $\mathcal{B}$ and on the choice of the sets $R^{*}$, although for simplicity our notation does not refect this dependence.

We will need the following result.

Theorem 2. Let $1<p \leq q<\infty$ and $0 \leq \alpha<1$, and let $M_{\alpha}$ be defined by (1.10), assuming that (1.9) holds. If there exists $r>1$ such that

$$
|R|^{\alpha-\frac{1}{p}} w\left(R^{*}\right)^{\frac{1}{q}}\left(\frac{1}{|R|} \int_{R} \sigma^{r} d x\right)^{\frac{1}{r^{\prime}}} \leq c
$$

for all $R \in \mathcal{B}$, then

$$
\left\|M_{\alpha} f\right\|_{q, w} \leq c\|f\|_{p, v}
$$

with a constant $c$ which is a multiple depending on $\alpha, n, p$, and $q$, but not on $\mathcal{B}$ or $f$, of the constant in (1.11).

We note that the condition

$$
|R|^{\alpha-1} w\left(R^{*}\right)^{\frac{1}{6}} \sigma(R)^{\frac{3}{p^{\prime}}} \leq c ; \quad R \in \mathcal{B},
$$

is necessary for (1.12) (even for the corresponding weak-type result), as can be seen by choosing $f=\chi_{R} \sigma$ in (1.12) and using a standard argument.

In case $R^{*}=R$ and $R$ is a cube, Theorem 2 is due to C. Pérez [P1], $[\mathrm{P} 2]$. Our proof will be modeled on ideas in [SW] and is given in Section 2 . 


\section{Proof of Theorem 2}

The proof of Theorem 2 uses some ideas from [SW]. The details which are either the same or nearly the same as ones there will be omitted.

Let $\mathcal{B}=\mathcal{B}\left(R_{\mathcal{B}}\right)$ be a family a rectangles $R$ as in the introduction, with associated sets $R^{*}$ which satisfy (1.9). Let $e_{1}, \ldots, e_{n}\left(e_{1}=1\right.$, say) bc the edgelengths of $R_{\mathcal{B}}$, and let $\mathcal{B}^{d y}$ be the corresponding grid of dyadic rectangles of the form

$$
\left[\frac{m_{1} e_{1}}{2^{j}}, \frac{\left(m_{1}+1\right) e_{1}}{2^{j}}\right] \times \cdots \times\left[\frac{m_{n} e_{n}}{2^{j}}, \frac{\left(m_{n}+1\right) e_{n t}}{2^{j}}\right]
$$

for $j, m_{1}, \ldots, m_{n}=0, \pm 1, \pm 2, \ldots$. Each rectangle in $\mathcal{B}^{d y}$ is also in $\mathcal{B}$. Define

$$
M_{\alpha}^{d y} f(x)=\sup _{\substack{R \in \mathcal{B}^{d y} \\ R^{3} \ni x}}|R|^{\alpha-1} \int_{R}|f(y)| d y,
$$

and for $z \in \mathbb{R}^{n}$, define.

$$
M_{\alpha}^{d y, z} f(x)=\sup _{\substack{R \in \mathcal{B}^{d y} \\(R+z)^{*} \ni x}}|R+z|^{\alpha-1} \int_{R+z}|f(y)| d y .
$$

Of course, $|R+z|=|R|$.

Lemma (2.1). If $1 \leq q \leq \infty$ and $w$ is a weight, then

$$
\left\|M_{\alpha} f\right\|_{q: w} \leq c \sup _{z \in \mathbb{R}^{n}}\left\|M_{\alpha}^{d y_{i} z} f\right\|_{q, w}
$$

with $\mathcal{C}$ depending on $\alpha$ and $n$ but not on $\mathcal{B}$ or $f$.

Proof: We argue as in $[\mathbf{W}]$ and $[\mathbf{S W}]$, and earlicr $[\mathbf{F S}]$. The important part of the argument is as follows. Fix $R \in \mathcal{B}$ and consider the collection of those $R_{1} \in \mathcal{B}^{d y}$ whose edgelengths are about twice those of $R$, respectively, and think of $\mathbb{R}^{n}$ as partioned into the union of such $R_{1}$. Of course, $\left|R_{1}\right| \approx|R|$ for each $R_{1}$ with constants of equivalence depending only on $n$. A simple geometric argument using translations shows that for each $R_{1}$,

$$
\left|\left\{z \in \mathbb{R}^{T_{L}}: R \subset R_{1}+z\right\}\right| \geq c\left|R_{1}\right|
$$

with $c>0$ depending only on $n$. Also, with $R$ still fixed, the sets $\{z \in$ $\left.\mathbb{R}^{n}: R \subset R_{1}+z\right\}$ are essentially disjoint for diflerent (nonoverlapping) $R_{1}$. Let

$$
E\left(R_{1}\right)=\left\{z \in \mathbb{R}^{n}: R \subset R_{1}+z, R^{*} \subset\left(R_{1}+z\right)^{*}\right\}
$$


and note by (1.9) that $E\left(R_{1}\right)$ is the same as the set $\left\{z \in \mathbb{R}^{n}: R \subset R_{1}+z\right\}$ above. Also if $x \in R^{*}$ and $z \in E\left(R_{1}\right)$, then

$$
|R|^{\alpha-1} \int_{R}|f(y)| d y \leq c\left|R_{1}+z\right|^{\alpha-1} \int_{R_{1}+z}|f(y)| d y \leq c M_{\alpha}^{d y_{1} z} f(x)
$$

since $\left|R_{1}+z\right|=\left|R_{1}\right| \approx|R|, R \subset R_{1}+z$ and $x \in\left(R_{1}+z\right)^{*}$. The constant $c$ depends only on $n, \alpha$.

The key points to observe are that if we denote $\Omega=\bigcup_{R_{1}} E\left(R_{1}\right)$, then the inequality between the first and third terms in (2.2) holds if $x \in R^{*}$ and $z \in \Omega$, that $\left|E\left(R_{1}\right)\right| \geq c\left|R_{1}\right|$ for each $R_{1}$, and that the $E\left(R_{1}\right)$ are essentially disjoint for different $R_{1}$. The rest of the proof then proceeds as in $[\mathbf{S W}]$ or $[\mathbf{W}]$, and is omitted.

To prove Theorem 2, it is enough by Lemma (2.1) to prove the analogue of (1.12) for cach $M_{\alpha}^{d y_{1} z} f$, with a constant independent of $z$. If we replace $f$ by $f \sigma$, this amounts to showing that

$$
\left\|M_{\alpha}^{d y, z}(f \sigma)\right\|_{q, w} \leq c\|f\|_{p, \sigma}
$$

with $c$ equal to a multiple depending only on $\alpha, n, p$ and $q$ of the constant in (1.11).

To prove (2.3), fix $z$ and $f \geq 0$, and for $k=0, \pm 1, \pm 2, \ldots$, let

$$
\Omega_{k}=\left\{x \in \mathbb{R}^{n}: M_{\alpha}^{d y, z}(f \sigma)(x)>2^{k n}\right\} .
$$

Then $x \in \Omega_{k}$ if and only if there exists $R \in \mathcal{B}^{d y}$ such that $x \in(R+z)^{*}$ and

$$
|R|^{\alpha-1} \int_{R+z} f \sigma d y>2^{k n} .
$$

In particular, if $R \in \mathcal{B}^{d y}$ and (2.4) holds then $(R+z)^{*} \subset \Omega_{k}$. Let $\left\{R_{j}^{k}\right\}_{j}$ be the maximal (with respect to inclusion) rectangles in $\mathcal{B}^{d y}$ which satisfy (2.4); their existence is assured if $f$ has compact support, which we may assume to be the case without loss of generality. By maximality, the $\left\{R_{j}^{k}+z\right\}_{j}$ are nonoverlapping for each $k$. Moreover, if $\bar{R}_{j}^{k}$ is the next largest dyadic rectangle containing $R_{j}^{k}$, then

$$
\left|\bar{R}_{j}^{k}\right|^{\alpha-1} \int_{\bar{R}_{j}^{k}+z} f \sigma d y \leq 2^{k n}
$$

by maximality, so that since $\left|\bar{R}_{j}^{k}\right|=2^{n}\left|R_{j}^{k}\right|$, we have

$$
2^{k n}<\left|R_{j}^{k}\right|^{\alpha-1} \int_{R_{j}^{k+z}} f \sigma d y \leq 2^{n(1-\alpha)} 2^{k n} .
$$


We claim that

$$
\Omega_{k}=\bigcup_{j}\left(R_{j}^{k}+z\right)^{*}
$$

We have already observed that each $\left(R_{j}^{k}+z\right)^{*}$ must lie in $\Omega_{k}$. On the other hand, if $x \in \Omega_{k}$ there is a dyadic $R$ with $x \in(R+z)^{*}$ such that (2.4) holds. Thus $R \subset R_{j_{0}}^{k}$ for some $j_{0}$ (since $R$ is maximal or not), and consequently $(R+z)^{*} \subset\left(R_{j_{0}}^{k}+z\right)^{*}$ by $(1.9)$. Hence, $x \in\left(R_{j_{0}}^{k}+z\right)^{*}$ and the claim follows.

By (2.6),

$$
\Omega_{k} \backslash \Omega_{k+1}=\bigcup_{j}\left[\left(R_{j}^{k}+z\right)^{*} \backslash \Omega_{k+1}\right]=\bigcup_{j} E_{j}^{k}
$$

where $E_{j}^{k}=\left(R_{j}^{k}+z\right)^{*} \backslash \Omega_{k+1}$. Thus

$$
\begin{aligned}
\left\|M_{\alpha}^{d y, z}(f \sigma)\right\|_{q, w}^{q} & =\int_{\mathbb{R}^{n}}\left[M_{c z}^{d y, z}(f \sigma)(x)\right]^{q} w(x) d x \\
& =\sum_{k} \int_{\Omega_{k} \backslash \Omega_{k+1}} \cdots \\
& \leq \sum_{k, j} 2^{(k+1) n q} w\left(E_{j}^{k}\right) \\
& \leq 2^{n q} \sum_{k, j}\left(\left|R_{j}^{k}\right|^{\alpha-1} \int_{R_{j}^{k}+z} f \sigma d y\right)^{q} w\left(E_{j}^{k}\right) \\
& \left.=\left.2^{n q} \sum_{k, j} w\left(E_{j}^{k}\right)|| R_{j}^{k}\right|^{\alpha-1} A\left(R_{j}^{k}+z\right)\right]^{q} . \\
\qquad & \quad\left(\frac{1}{A\left(R_{j}^{k}+z\right)} \cdot \int_{R_{j}^{k}+z} f \sigma d y\right)^{q},
\end{aligned}
$$

where for a rectangle $R$,

$$
A(R)=|R|^{\frac{1}{r^{r}}}\left(\int_{R} \sigma^{r} d y\right)^{\frac{1}{r}}
$$

We estimate the last sum by using hypothesis (1.11) for the rectangles $R_{j}^{k}+z$ and the fact that $E_{j}^{k} \subset\left(R_{j}^{k}+z\right)^{*}$, obtaining that

$$
\left\|M_{\alpha}^{d y, z}(f \sigma)\right\|_{q, w}^{q} \leq 2^{n q} c^{q} \sum_{k, j} A\left(R_{j}^{k}+z\right)^{\frac{q}{p}}\left(\frac{1}{A\left(R_{j}^{k}+z\right)} \int_{R_{j}^{k}+z} f \sigma d y\right)^{q},
$$


where $c$ is the constant in (1.11).

The remainder of the proof is based on using the next lemma to estimate the sum in (2.7).

Lemma 2.8. Let $\left\{R_{i}\right\}_{i \in I}$ be a collection of rectangles from a fixed dyadic grid (e.g., from $\mathcal{B}^{d y}+z$ for fixed $z$ ), let $\beta \geq 1$, and let $\left\{a_{i}\right\}_{i \in I}$ be positive numbers which satisfy

(i) $\sigma\left(R_{i}\right) \leq c_{0} a_{i}$

(ii) $\sum_{j: R_{j} \subset R_{i}} a_{j}^{\beta} \leq c_{0} a_{i}^{\beta}$

for each $i$, with $c_{0}$ independent of $i$. Then if $1<p<\infty$ and $q=\beta p$,

$$
\left[\sum_{i \in I} a_{i}^{\beta}\left(\frac{1}{a_{i}} \int_{R_{i}}|f| \sigma d y\right)^{q}\right]^{\frac{3}{q}} \leq c\|f\|_{p, \sigma}
$$

with $c$ depending on $c_{0}, p$ and $q$, but not on $f$ or the particular grid.

The proof is virtually the same as that of Lemma (2.10) of [SW], which deals with the case of dyadic cubes, and is therefore omitted.

If we apply Lemma (2.8) to the sum in (2.7) and note that $\sigma(R) \leq$ $A(R)$ by Hölder's inequality, we immediately obtain (2.3) from (2.7) if we verify

$$
\sum_{k, g: R_{j}^{k} \subset R_{l}^{m}} A\left(R_{j}^{k}+z\right)^{q / p} \leq c A\left(R_{l}^{m}+z\right)^{q / p}
$$

for each $R_{l}^{m}$ and $0<p \leq q<\infty$, with $c$ independent of $l, m$ and $z$. We argue as in the proof of (2.11) in [SW]. Using the simple inequality $\sum a_{i}^{q / p} \leq\left(\sum a_{i}\right)^{q / p}, q \geq p, a_{i} \geq 0$, we may prove just the case $q=p$. If $R_{j}^{k}$ is a proper subset of $R_{l}^{m}$ then

$$
2^{m n}<\left|R_{l}^{m}\right|^{\alpha-1} \int_{R_{l}^{m}+z} f \sigma d y \leq 2^{k n},
$$

where the second inequality follows from the maximality of $R_{j}^{k}$. Therefore, we must have $k \geq m$ in (2.9), and we may rewrite the left side of (2.9) (with $q / p=I$ ) as

$$
\sum_{k=m}^{\infty} \sum_{j: R_{j}^{k} \subset R_{i}^{m}} A\left(R_{j}^{k}+z\right)
$$


By Hölder's inequality and the definition of $A(R)$, the inner sum in (2.10) is at most

$$
\left(\sum_{j: R_{j}^{k} \subset R_{l}^{m}}\left|R_{j}^{k}\right|\right)^{\frac{1}{r^{\prime}}}\left(\sum_{j: R_{j}^{k} \subset R_{i}^{m}} \int_{R_{j}^{k}+z} \sigma^{r} d y\right)^{\frac{1}{r}} .
$$

Here, since $\left\{R_{j}^{k}+z\right\}_{j}$ are nonoverlapping for fixed $z$, the second factor is at most $\left(\int_{R_{t}^{n}+z} \sigma^{r} d y\right)^{\frac{1}{r}}$. Also, for the first factor, by the first inequality $(2.5)$,

$$
\begin{aligned}
\sum_{j: R_{j}^{k} \subset R_{l}^{m}}\left|R_{j}^{k}\right| & \leq \sum_{\ldots}\left(2^{-k n} \int_{R_{j}^{k}+z} f \sigma d y\right)^{\frac{1}{1-\alpha}} \\
& \leq\left(\sum_{\ldots} 2^{-k n} \int_{R_{j}^{k}+z} f \sigma d y\right)^{\frac{1}{1-\alpha}} \text { since } \frac{1}{1-\alpha} \geq 1 \\
& \leq\left(2^{-k n} \int_{R_{l}^{m}+z} f \sigma d y\right)^{\frac{1}{1-\alpha}} \\
& \leq\left(2^{-k n}\left|R_{l}^{m}\right|^{1-\alpha} 2^{n(1-\alpha)} 2^{m n}\right)^{\frac{1}{1-\alpha}}
\end{aligned}
$$

by the second inequality (2.5),

$$
=2^{n} 2^{(m-k) n /(1-\alpha)}\left|R_{l}^{m}\right| \text {. }
$$

Combining estimates, we see $(2.10)$ is at most

$$
\sum_{k=m}^{\infty}\left(2^{n} 2^{(m-k) n /(1-\alpha)}\left|R_{i}^{m}\right|\right)^{\frac{1}{r^{\prime}}}\left(\int_{R_{l}^{m}+z} \sigma^{r} d y\right)^{\frac{1}{r}}=c A\left(R_{i}^{m}+z\right),
$$

as desired. This proves (2.9), and also completes the proof of Theorem 2 .

\section{Proof of Theorem 1}

We now show how Theorem 1 implies Theorem 2. In fact, we will prove a more general result based on the following fact from [CWW]. Let $S$ be a set in $\mathbb{R}^{n}$ which is starlike with respect to the origin, i.e., except at most for a set of measure 0 on the boundary, $S=\{r \theta: \theta \in$ $\left.S^{n-1}, 0<r \leq \rho(\theta)\right\}$, where $\rho$ is the boundary function of $S$. 
Lemma 3.1. If $S$ is starlike with respect to the origin, there exist rectangles $\left\{R_{j}\right\}$ (with varying orientations) such that each $R_{j}$ contains the origin on its major axis, $S \subset \bigcup_{j} R_{j}$, and $\sum\left|R_{j}\right| \leq C|S|$ for some $C>0$.

For more facts concerning such covers, we refer to [CWW].

Given a starlike $S$ with respect to the origin, fix a cover $\left\{R_{j}\right\}$ as above. Given a bounded set $E$, define $R_{j}^{*}$ to be a rectangle containing both $E$ and $R_{j}$. Also, for each $j$, let $\mathcal{B}_{j}$ denote the collection of all dilates and translates of $R_{j}$, and if $R \in \mathcal{B}$, say $R=z+t R_{j}$, define $R^{*}=z+t R_{j}^{*}$. The pairs $\left(R, R^{*}\right), R \in \mathcal{B}_{j}$, then satisfy $(1,9)$.

Theorem 3. Let $0 \leq \mu<n, 1<p \leq q<\infty, S$ be a starlike set with respect to the origin, and $E$ be a bounded set. With the notation above, assume there exists $r>1$ so that

$$
|R|^{\frac{\mu}{n}-\frac{1}{p}} w\left(R^{*}\right)^{\frac{1}{q}}\left(\frac{1}{|R|} \int_{R} v^{-\frac{r}{p-1}} d x\right)^{\frac{1}{r_{p}^{\prime}}} \leq C_{R_{j}}\left|R_{j}\right|^{\frac{\mu}{n}-1}
$$

for all $R \in \mathcal{B}_{j}$, and that $\sum C_{R_{j}}<\infty$. Then

$$
\left\|\mathcal{M}_{S, E, \mu} f\right\|_{q, w} \leq c\|f\|_{p, v} .
$$

Before giving the proof, we note that Theorem 3 includes Theorem 1 by picking $S=S_{\gamma}, E=Q_{1}$ and $R_{j}=\delta_{a} Q_{1}$ for $a=2^{j}, j=1,2, \ldots$, i.c., $R_{2}$ in the notation of the introduction. Recall that in the introduction $R_{a}^{*}$ is the smallest rectangle containing both $R_{a}$ and $Q_{1}$. The requirement in Theorem 3 that $\sum C_{R_{j}}<\infty$ then amounts to (1.5).

To prove Theorem 1, write

$$
\begin{aligned}
\mathcal{M}_{S, E, \mu} f(x)= & \sup _{\substack{z \in \pi^{n}, t>0 \\
x \in z+t E}} t^{\mu-n} \int_{z+t S}|f(y)| d y \leq \\
& \leq \sum_{j}\left|R_{j}\right|^{1-\frac{\mu}{n}}\left(\sup _{\substack{z \in \mathbb{R}^{n}, t>0 \\
x \in z+t E}}\left|t R_{j}\right|^{\frac{\mu}{n}-1} \int_{z+t R_{j}}|f(y)| d y\right)
\end{aligned}
$$

since $\left|t R_{j}\right|=t^{n}\left|R_{j}\right|$ and $S \subset \bigcup_{j} R_{j}$. Since $E \subset R_{j}^{*}$, we have $z+t E \subset$ $z+t R_{j}^{*}=\left(z+t R_{j}\right)^{*}$ by definition. Thus, in the notation of Theorem 2,

$$
\mathcal{M}_{S, E, \mu} f(x) \leq \sum_{j}\left|R_{j}\right|^{1-\frac{\mu}{n}} M_{\frac{E}{n}, j} f(x)
$$


where $M_{\frac{\mu}{n}, j}$ is the maximal function (1.10), using the rectangles in $\mathcal{B}_{j}$. Therefore,

$$
\begin{aligned}
\left\|M_{S, E, \mu} f\right\|_{q, w} & \leq \sum_{j}\left|R_{j}\right|^{1-\frac{\mu}{n}}\left\|M_{\frac{\mu}{n}, j} f\right\|_{q, w} \\
& \leq c \sum_{j}\left|R_{j}\right|^{1-\frac{\mu}{n}} C_{R_{j}}\left|R_{j}\right|^{\frac{\mu}{n}-1}\|f\|_{p, v} \\
& =c\left(\sum C_{R_{j}}\right)\|f\|_{p, v}
\end{aligned}
$$

by (3.1) and Theorem 2. This completes the proof of Theorem 3.

\section{References}

[Ca] C. P. CALdERóN, Differentiation through starlike sets in $\mathbb{R}^{m}$, Studia Math. 48 (1973), 1-13.

[CWW] S. Chanillo, D. K. Watson and R. L. Wheedfn, Some integral and maximal operators related to starlike sets, Studia Moth. (to appear).

[Cor] A. Córdoba, Maximal functions, covering lemmas and Fourier multipliers, Proc. Symp. Pure Math. 35, part 1 (1979), A.M.S., $29-50$.

[FS] C. FEFfERman and E. M. Stein, Some maximal inequalities, Amer. J. Math. 93 (1971), 107-115.

[J] B. JAWERTH, Weighted inequalities for maximal operators: linearization, localization, and factorization, Amer. J. Math. 108 (1986), 361-414.

[P1] C. PÉREZ, On sufficient conditions for the boundedness of the Hardy-Littlewood maximal operator between weighted $L^{p}$-spaces with different weights (to appear).

Added in Proof:

For some different results related to Theorem 2, see the articles

(1) F. J. Ruiz and J. L. Torrea, Weighted norm inequalities for a general maximal operator, Atk. Mat. 26 (1988), 327-340.

(2) Pan Wenjie, "Weighted norm inequalities for certain maximal operators with appraach regions", Lecture Notes in Math. 1494, Springer, 1992, 169 175.

(3) A. Sánchez-Colomer and J. Soria, Weighted norm incqualities for general maximal operators and approach regions, Preprint. 
[P2] C. PÉRE2, Two weighted inequalities for potential and fractional type maximal operators, Indiana U. Math. J. (to appear).

[P3] C. PÉREz, A remark on weighted inequalities for general maximal operators, Proc. Amer. Math. Soc. (to appear).

[SW] E. SAWYER AND R. L. WheEden, Woighted inequalities for fractional integrals on Euclidean and homogeneous spaces, Amer. J. Math. 114 (1992), 813874.

[W] R. L. WheEden, A characterization of some weighted norm inequalities for the fractional maximal function, Studia Math. (to appear).

Department of Mathematics

Rutgers University

New Brunswick, NJ 08903

U.S.A.

Rebut el 29 de Juny de 1993 